\title{
NATURAL FIBRE TEXTILE NANO-LEVEL SURFACE MODIFICATION
}

\author{
Svetlana Vihodceva ${ }^{2}$, Jānis Barloti ${ }^{1}$, Silvija Kukle², Gunta Zommere ${ }^{2}$ \\ 1- Riga Technical University, Faculty of Electronics and Telecommunications \\ Azenes St. 12, Riga, LV 1048, Latvia \\ 2- Riga Technical University, Department of Textile Technologies and Design \\ Azenes St. 14/24, Riga, LV 1048, Latvia \\ Ph.: + (371) 67089816, fax: + (371) 67089816, e-mail: Svetlana.Vihodceva@rtu.lv
}

\begin{abstract}
A variety of applications requires production of textile materials with specially designed surface properties. Surface coating by metallic materials have attracted a lot of attention due to their special surface properties, such as UV-absorbsion, antimicrobial, anti-fungicidal and ect. The paper describes the process of vacuum evaporation and magnetron sputtering of copper layers on cotton textile materials and analysis of the metal coated textile surface morphology by laser laboratory complex. The textile samples have been finished by magnetron sputtering and vacuum evaporation technologies and tested for bonding strength, light reflection and light passing properties. The paper presents and discusses the results of the experiment.
\end{abstract}

Keywords: copper, cotton, magnetron sputtering, vacuum evaporation, textile.

\section{Introduction}

Nanotechnology brings us a step closer towards materials with specific individual properties and represents a shift away from the catalogue range of standard materials. Surfaces emancipate themselves from the underlying material, developing clearly defined additional functions that can differ fundamentally from the substrate material. The goal of nanotechnology is a consistent usage of the minimum amount of raw material and energy, from an economic and ecological point of view, in the long term "nano" is a winning factor. The use of nanotechnology offers ecological and economic advantages for energy efficiency and the conservation of resources. [1]

It is important to take a life-cycle approach to evaluate the environmental as well as human health impacts at each stage of a nano-enabled product's life cycle (Fig.1.) before arriving at any conclusions regarding the product's potential environmental benefits or drawbacks.

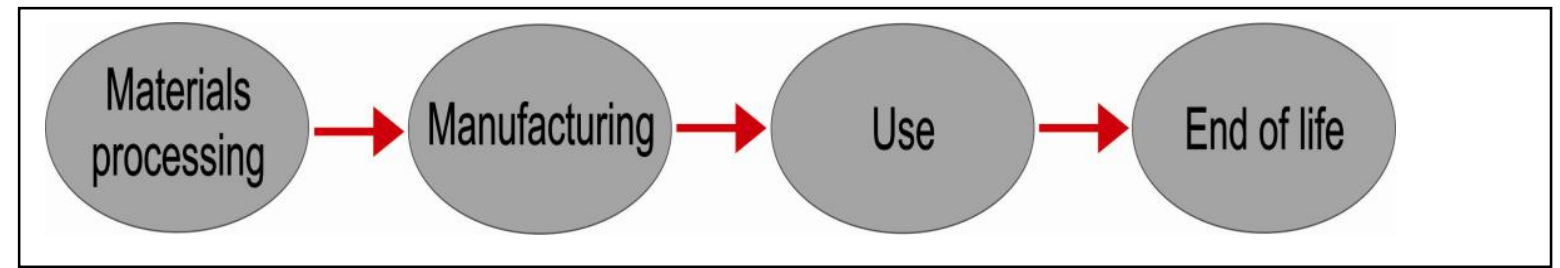

Fig. 1. Product life-cycle

Manufacturing process requires energy, but creation of nano-level textile coatings needs a shorten process time which reduces energy consumption. At present manufacturing processes for the production of nanomaterials are not standardized, but are in an evolutionary stage, changing constantly. The techniques for textile surface modification generally are grouped in two major categories: chemical and physical. Physical methods are based on usage of nonchemical forces to control deposition of functional material on the textile surface. [2] Having the ability to control the surfaces of a natural fibers offers great rewards that go far beyond pure economics as natural fibers are renewable and biodegradable resources. [3] 
At present consumers are becoming increasingly aware of the hygienic life style and there is a necessity and expectation for a wide range of textile products finished with biocide properties. One way to avoid the microbial degradation of textile fibres, limit the incidence of bacteria and fungi, and protect users, is the treatment of textiles with antimicrobial agents, such as silver and copper. Man has been exploiting the natural antimicrobial properties of copper since the dawn of civilization. It has been demonstrated clearly in many scientific studies conducted over several decades that copper reduces contamination of the most toxic species of bacteria, fungi and viruses, and inhibits its growth.

Copper is considered to be safe for humans, as demonstrated by the widespread and prolonged use by women of copper intrauterine devices (IUDs). [4] Animal studies have demonstrated that copper fibers do not possess skin sensitizing properties. These findings are in accordance with the very low risk of adverse skin reactions associated with copper. $[5,6]$ In contrast to the low sensitivity of human tissue (skin or other) to copper, microorganisms are extremely susceptible to copper [7,8]: copper surface kills over 99.9\% of bacteria (Escherichia coli, Enterobacter aerogenes, MRSA, Pseudomonas aeruginosa, Staphylococcus aureus) for 24 hours. [9]

\section{Materials and methods}

Object of investigation. Commercial woven $100 \%$ plain wave cotton fabric with surface linear density $276.19 \mathrm{~g} / \mathrm{m} 2$ from yarns of linear density 9.2 tex has been used in the experiment. The thickness of the textile fabric is approximately $0.25 \mathrm{~mm}$; the measurement was taken by the textile fabric thickness tester ("TH-25", "Zapadpribor", Ukraine).

Pre-treatment of textile fabric. The ability of a particular construction textile fabric to hold treatment depends on the level of cleanness of its surface i.e. oils, waxes, sizes applied in yarn and fabric manufacturing processes, dust and dirt particles contaminant the surface of the fabric must be removed to provide good interfacial contact between fiber surface and deposited product.

The previous research evince that evenness of copper coating and copper concentration amount on sample of materials deviated from physical pre-treatment time in $80 \%$ acetone solution, the results of the experiment show that 5 minutes is the optimal immersing time in acetone solution. [10] It means that after immersing in acetone solution for 5 minutes the copper was well distributed on the fabric surface and more copper was deposited on the surface. [10]

Three different methods to increase adhesion force are under inspection: 1) to remove dust and organic matter from the surface of woven cotton fabric the samples were washed at temperature $90^{\circ} \mathrm{C}$ with detergent without optical brighteners, 2) after samples were immersed in $80 \%$ acetone solution at room temperature for 5 minutes, and were washed twice with distilled water (ISO 9001, ISO 14001), the drying step was carried out on a horizontal surface, 3) surface modification by plasma.

Surface modification by plasma. Low temperature plasma treatment is a quite new technology for the natural fiber textiles; plasma is essentially a dry process providing modification of the top nanometer surface. [11] The advantages of plasma technology are its potential environmental friendliness and energy conservation benefits in developing highperformance materials. [11] It offers many advantages against wet techniques: there are no harmful chemicals, wet processes, wastewater, and mechanical hazards to textiles, etc. In addition it has a specific impact on the surface and gives surface some properties that cannot be obtained by conventional techniques. [12]

The plasma treatment as activation method of the surface of cotton fabric is used to improve copper adhesion to the fabric surface followed by the deposition of the metallic layers by 
vacuum evaporation technology. The results of our previous research evince that plasma activation of the materials surface followed by the deposition of the metallic layer by vacuum evaporation positively influences copper concentration on the surface and copper coat evenness. [13]

In vacuum evaporation technology, plasma treatment and coating with copper, is a two-stage process. It is possible significantly simplify this process, by the method of magnetron sputtering, which unites the two processes mentioned above in one. This is a quick, ecological process, practically without yielding any waste and characterized by high repeatability, stability and efficiency.

Vacuum evaporation technology. In high-vacuum evaporation $\left(1 * 10^{-4}-5^{*} 10^{-5} \mathrm{~mm} \mathrm{Hg}\right)$ chamber „UVN - 2U" alternating current electrical heating is used to melt, gasify and evaporate copper. Then the copper vapour travels to the surface of textile material and gradually cools. Vacuum conditions prevent the collision of the evaporated functional particles with the background gas or other unwanted particles. As "UVN-2U" uses a pointsource to evaporate the coating material and copper melting and evaporation temperature is rather high $\left(>1400{ }^{\circ} \mathrm{C}\right)$ for cotton fibres, to protect cotton fibres from high temperature is used special protective screen. Short exposure time ( 1 to 2 seconds) is sufficient to get a thin copper layer without destruction of substrate material from natural fibres. Experimental results show that during one exposition (1 second) $60-70 \mathrm{~nm}$ thick film of copper is formed on the surface of cotton fabric sample. [10]

Magnetron sputtering technology. Magnetron sputtering is a plasma coating process whereby sputtering material is ejected due to bombardment of ions to the target surface. The vacuum chamber of the PVD coating machine is filled with an inert gas - argon. By applying a high voltage, a glow discharge is created, resulting in acceleration of ions to the target surface and a plasma coating. The argon-ions will eject sputtering materials from the target surface (sputtering), resulting in a sputtered coating layer on the products in front of the target.

Non-contact method of surface examination. In order to get a detailed insight into the change of the surface properties and develop a tool for comparative analysis the surface of the sample has been examined with the laboratory laser complex. The experiment has been carried out by the laser beam, changeable angles between falling and reflected light beams were $-20^{\circ},-15^{\circ}, 5^{\circ}, 10^{\circ}, 15^{\circ}$, and $20^{\circ}$ degrees. Measurements of the reflected light, that show how changes the textile surface morphology after it coating with copper. [10, 13]

The passing light has been measured by laboratory laser complex that makes it possible to determine the evenness level of the copper layer and the copper layer changes after exploitation impacts (Fig.2.).

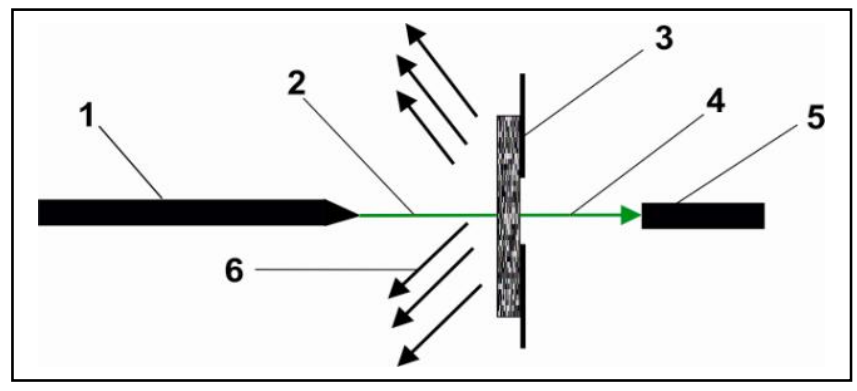

Fig.2. Laboratory laser complex for the measuring of passing through light:

1 - laser; 2 - incident laser beam; 3 - textile sample on holder; 4 -passing laser beam; 5 -detector of passing light; 6 - scattered light 


\section{Results and discussion}

Copper particles can be deposited onto cotton fabrics by vacuum evaporation and magnetron sputtering technologies.

The results of the research show that vacuum evaporation has such disadvantage as low bonding strength of functional coating with the substrate surface. $[10,13]$ In our experiments we have achieved the twofold increase of surface adhesion after pret-treatment immersing in $80 \%$ acetone solution for $5 \mathrm{~min}$ and surface modification by low pressure plasma, but still exploitation of textile have a considerable influence on the copper layer. $[10,13]$

The samples have been washed in order to observe the see effects of exploitation processes on the copper coated textile samples finished by magnetron sputtering technology. Before washing and after samples of all washing times were measured passing through and reflected coherent light. The received passing light measurement results show, that light passing of the textile material samples covered with copper is only $48 \%$ in comparison to the light passing of the uncovered materials, after the first washing it is $60 \%$, and after the second washing $63 \%$.

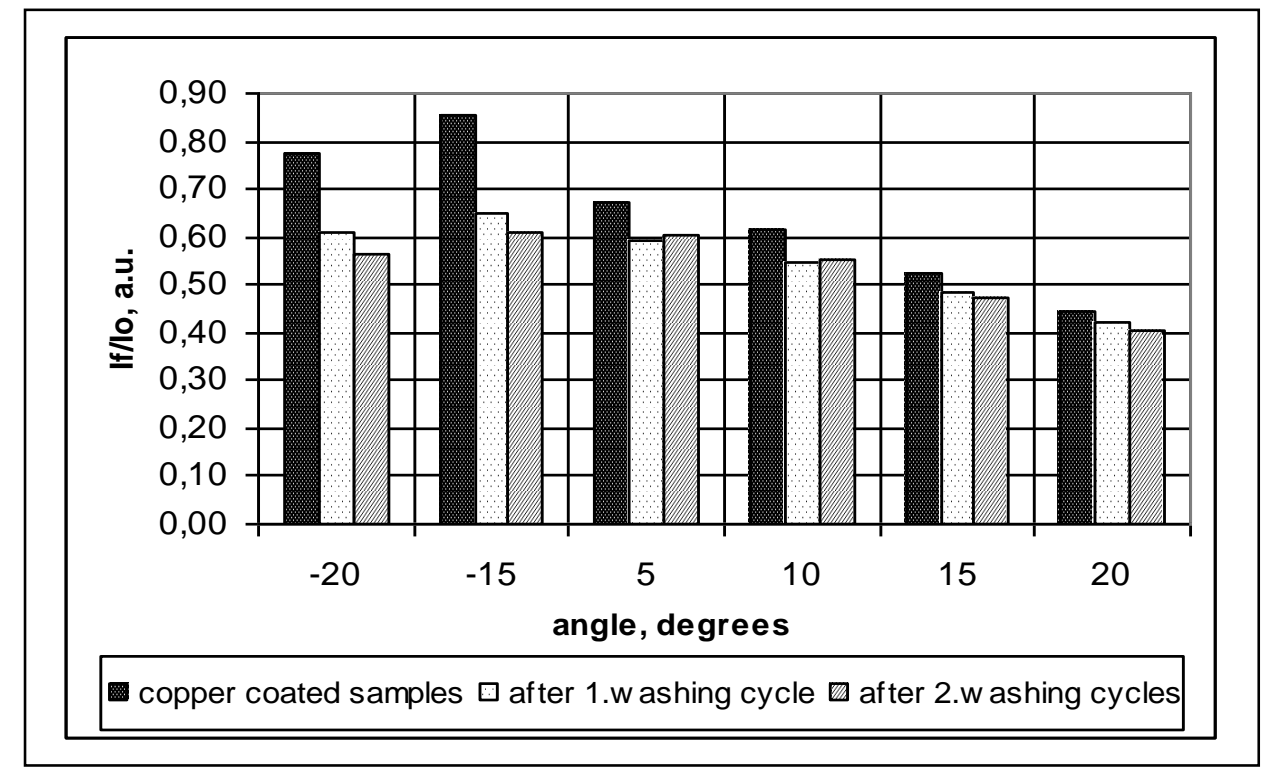

Fig.3. Reflected light of coated samples before and after washing

(a.u. - the level of light intensity, rationed to maximum)

The graph (Fig.3.) describes the measurement results of the reflected light by non-contact method of surface examination show similar results as passing light measurements. The graphs of Fig. 3 shows as well that the amount of reflected light decrease rapidly after the first washing, especially for small angles. It means that a part of the deposited copper was washed out during the first washing procedure and regular light reflection of the copper film diminished. The graph also shows that the effect of the second washing is insignificant; i.e. the unsteady copper particles were washed out during first cycle, and adhesion of the remaining particles is sufficient to resist repeating washing processes cycles.

The results of the experiment evince that reflected and passing light show that measurements of reflected and passing light can be applied to trace the metal covered fabric surface changes from exploitation impacts. 


\section{Summary}

Application of nanotechnology offers ecological and economic advantages for energy efficiency and the conservation of resources. Magnetron deposition of nanolevel metal coatings on the textile surface reduces energy consumption in comparison with expenditures of the resources in traditional textiles finishing processes.

Vacuum evaporation and magnetron sputtering are physical methods based on application of non-chemical forces for deposition control of functional material on the textile surface.

Laser laboratory complex measurements of reflected and passing through covered material light can be applied to trace the unevenness of deposited metal film on the covered fabric surface and its changes from exploitation impacts.

Ability to add additional properties to the surfaces of natural fibers and textiles offers not only environmental and economic rewards, but solve problems connected with global resources shortening as natural fibers are yearly renewable and fully biodegradable resources.

\section{References}

1. Leydecker S. Nanomaterials in architecture, interior architecture and design. Birkhäuser Verlag AG, ISBN978-3-7643-7995-7, 2008.

2. Q. Wei, Y. Xu and Y. Wang. Textile Surface Functionalization by Physical Vapour Deposition. In Surface Modification of Textiles, Woodhead Publishing Ltd., ISBN 978-1-84569-419-7, Cornwalk UK, 2009.

3. Brown, P.J.; Stevens, K.. Nanofibres and nanotechnology in textiles, Woodhead Publishing Series in Textiles No.67., ISBN 1-84569-105-9, UK, 2007.

4. Hubacker, D. et al: Use off copper intrauterine devices and the risk of tubal infertility among nulligravid women, Engl.J Med, 345(8): 561-567, (2001)

5. Borkow, G.; Gabbay, J.: Putting copper into action: copper-impregnated products with potent biocidal activities, FASEB J., 18(14): 1728-1730(2004)

6. Hostynek, J.J.; Maibach, H.I.: Copper hypersensitivity: dermatologic aspects - an overview, Copper and the skin, CRC Press, ISBN 0849395321, 9780849395321, (2006)

7. Borkow, G.; GABBAY, J.: Using Copper Oxide in Medical Devices and Textiles To Fight DiseaseEffective, Inexpensive and Feasible, Cupron Inc., POD 10973, Greensboro NC 27404, USA

8. Dollwet, H.H.A.; Sorenson, J.R.J.: Trace Elements in Medicine, Historic uses of copper compounds in medicine, Vol. 2, No., (1985)

9. Protocol: Test Method for residual Self-Sanitizing Activity of Copper Alloy Surfaces, United States Enviromental Protection Agency, Available from http://www.epa.gov/oppad001/regpolicy.htm

10. Kukle S., Vihodceva S. Functionalisation of cotton textiles with metals. In: Book of proceedings of the 5th International Textile, Clothing \& Design Conference, Magic World of Textiles. Croatia, 2010. - 335-339 p.

11. Wei Q.: Surface modification of textiles, Woodhead Publishing Series in Textiles No.97., ISBN 978-184569-419-7, UK, 2009.

12. Kutlu, B.; Cireli, Dr.A.: Plasma technology in textile processing, Czech Textile Research Conference, 2004

13. Kukle S., Vihodceva S. Application of vacuum evaporation to obtain natural fiber textile products ultra thin metallic coatings. In: Book of abstracts 41st Symposium on Novelties in Textiles, 5ts Symposium on Novelties in Graphics. Slovenia 2010. - 413-419 p. 